GLOBAL JOURNAL OF MATHEMATICAL SCIENCES VOL. 11, NO. 1\&2, 2012: 27-34

\title{
FORECASTING IN ONE-DIMENSIONAL AND GENERALIZED INTEGRATED AUTOREGRES SIVE BILINEAR TIME SERIES MODELS
}

\section{J. F. OJO}

\begin{abstract}
In this paper, forecast of one-dimensional integrated autoregressive bilinear is compared with forecast of generalized integrated autoregressive bilinear model. We describe the method for estimation of these models and the forecast. It is also pointed out that for this class of non-linear time series models; it is possible to obtain optimal forecast. The estimation technique is illustrated with respect to a time series, and the optimal forecast of these time series are calculated. A comparison of these forecasts is made using the two models under study. The mean square error for forecast in generalized integrated autoregressive bilinear model is smaller than the mean square error for forecast in one-dimensional integrated autoregressive bilinear model. Though the two models are adequate for forecast when compared with the real series but forecast with generalized integrated autoregressive bilinear model is more adequate.
\end{abstract}

KEY WORDS: Optimal Forecast, Non-Linear Time Series Models, Bilinear Models, Estimation Technique, Mean Square Error.

\section{INTRODUCTION}

The bilinear time series models have attracted considerable attention during the last years. An overview of models and their application can be found in Subba Rao (1981), Pham and Tran (1981), Gabr and Subba Rao (1981), Rao et al. (1983), Liu (1992), Cathy (1997), Gonclaves et al. (2000), Shangodoyin and Ojo (2003), Wang and Wei (2004), Boonaick et al. (2005), Doukhan et al. (2006), Drost et al. (2007), Usoro and Omekara (2008) and Ojo( 2009). The bilinear modes studied by the above researchers could not achieve stationary for all nonlinear series. Ojo (2011) proposed one-dimensional integrated autoregressive bilinear time series model that could achieve stationary for all non linear time series. Also, Ojo and Shangodoyin (2010) proposed generalized integrated autoregressive bilinear time series model that could achieve stationary for all non linear time series.

Forecasting connote an attempt to see into the future. There are two words, which are used to denote numerical forecasting methods namely forecasting, and prediction. Forecasting is the process of estimation in unknown situations. Prediction is a similar, but more general term, and usually refers to estimation of time series, cross-sectional or longitudinal data. Forecasting is commonly used in discussion of time-series data. Therefore forecasting is a powerful useful instrument in planning and making a wise decision about future. As a result of feature of stationary for all non linear series in one dimensional and generalized integrated bilinear model we shall attempt to study optimal forecast using these two models and see the one that perform better.

\section{Generalized and One-Dimensional Integrated Autoregressive Bilinear Time Series Models}

We define generalized integrated autoregressive bilinear time series model as follows:

$\psi(B) X_{t}=\phi(B) \nabla^{d} X_{t}+\sum_{k=1}^{r} \sum_{l=1}^{s} b_{k l} X_{t-k} e_{t-l}+e_{t}$, denoted as GBL (p, d, 0, r, s)

where $\phi(B)=1-\phi_{1} B-\phi_{2} B^{2} \ldots \ldots-\phi_{p} B^{p}$ and

$$
X_{t}=\psi_{1} X_{t-1}+\ldots \ldots+\psi_{p+d} X_{t-p-d}+b_{11} X_{t-1} e_{t-1}+\ldots \ldots . .+b_{r s} X_{t-r} e_{t-s}+e_{t}
$$

$\phi_{1}, \ldots, \phi_{p}$ are the parameters of the autoregressive component; $b_{11}, \ldots \ldots \ldots, b_{r s}$ are the parameters of the non-linear component; and $d$ is the degree of consecutive differencing required to achieve stationary.

J. F. Ojo, Department of Statistics, University of Ibadan, Ibadan, Nigeria. 
We define one-dimensional integrated autoregressive bilinear time series models as follows:

$\psi(B) X_{t}=\phi(B) \nabla^{d} X_{t}+\left(\sum_{k=1}^{r} b_{k 1} X_{t-k}\right) e_{t-1}+e_{t}$, denoted as BL (p, d, 0, r, 1),

where $\phi(B)=1-\phi_{1} B-\phi_{2} B^{2} \ldots \ldots-\phi_{p} B^{p}$ and

$$
X_{t}=\psi_{1} X_{t-1}+\ldots \ldots+\psi_{p+d} X_{t-p-d}+\left(\sum_{k=1}^{r} b_{k 1} X_{t-k}\right) e_{t-1}+e_{t}
$$

$\phi_{1}, \ldots \phi_{p}$ are the parameters of the autoregressive component; $b_{11}, \ldots \ldots . ., b_{r 1}$ are the parameters of the nonlinear component and $d$ is the degree of consecutive differencing required to achieve stationary.

\section{Model Estimation}

The estimation of the models are similar, we shall report the estimation of generalized type since $m_{i}=1,2,3 \ldots ., s$ for the generalized case include $m_{i}=1$ the one dimensional case. Suppose that $X_{t}$ are generated by equation (1), the sequence of random deviates $\left\{e_{t}\right\}$ could be determined from the relation

$e_{t}=X_{t}-\psi_{1} X_{t-1}-\ldots \ldots . . .-\psi_{p+d} X_{t-p-d}-\sum_{k=1}^{r} \sum_{l=1}^{s} b_{k l} X_{t-k} e_{t-l}$

To estimate the unknown parameters in equation (3), we make the following assumptions:

(i) The errors $\left\{e_{t}\right\}$ are independent and identically distributed with mean zero and variance $\sigma^{2}$ with finite kurtosis.

(ii) The values of $\left|\psi^{\prime} s\right|<1$ and $\left|b_{k l}{ }^{\prime} s\right|<1$ ensure that invertibility condition required of the bilinear process is satisfied. For details see (Ojo and Shangodoyin, 2010).

Thus maximizing the likelihood function is equivalent to minimizing the function $Q(G)$, which is as follows:

$$
Q(G)=\sum_{i=m}^{n} e_{t}^{2}
$$

with respect to the parameter $G^{\prime}=\left(\psi_{1}, \ldots ., \psi_{p} ; B_{11}, \ldots ., B_{r s}\right)$

Then the partial derivatives of $Q(G)$ are given by

$$
\begin{aligned}
& \frac{d Q(G)}{d G_{i}}=2 \sum_{t=m}^{n} e_{t} \frac{d e_{t}}{d G_{i}} \quad(\mathrm{i}=1,2, \text { é .., R }) \\
& \frac{d^{2} Q(G)}{d G_{i} d G_{j}}=2\left(\sum_{t=m}^{n} e_{t} \frac{d e_{t}}{d G_{i}} \frac{d e_{t}}{d G_{j}}+\sum_{t=m}^{n} e_{t} \frac{d^{2} e_{t}}{d G_{i} d G_{j}}\right)
\end{aligned}
$$

where these partial derivatives of $e_{t}$ satisfy the recursive equations

$$
\begin{aligned}
& \frac{d e_{t}}{d \psi_{i}}+\sum_{j=1}^{s} W_{t}(t) \frac{d e_{t-j}}{d \psi_{i}}=X_{t-i} \text { if } \mathrm{i}=1,2, \text { é , } \mathrm{p} \\
& \frac{d e_{t}}{d B_{k m i}}+\sum_{j=1}^{s} W_{j}(t) \frac{d e_{t-j}}{d B_{k m i}}=-X_{t-k} e_{t-m} \quad\left(\mathrm{k}=1,2, \text { é }, \mathrm{r} ; \mathrm{m}_{\mathrm{i}}=1,2, \text { é ,s }\right)
\end{aligned}
$$




$$
\begin{aligned}
& \frac{d^{2} e_{t}}{d \psi_{i} d \psi_{i}^{\prime}}+\sum_{j=1}^{s} W_{j}(t) \frac{d^{2} e_{t-j}}{d \psi_{i} d \psi_{i}^{\prime}}=0\left(\mathrm{i}, \mathrm{i}^{\prime}=0,1,2, \text { é }, \mathrm{p}\right) \\
& \frac{d^{2} e_{t}}{d \psi_{i} d B_{k m i}}+\sum_{j=1}^{s} W_{j}(t) \frac{d^{2} e_{t-j}}{d B_{k m i} d \phi_{i}}+X_{t-k} \frac{d^{2} e_{t-m i}}{d \psi_{i}}=0 \\
& \frac{d^{2} e_{t}}{d B_{k m i} d B_{k m i}^{\prime}}+\sum_{j=1}^{s} W_{j}(t) \frac{d^{2} e_{t-j}}{d B_{k m i} d B_{k m i}^{\prime}}+X_{t-k}^{\prime} \frac{d^{2} e_{t-m i}}{d B_{k m i}}=-X_{t-k} \frac{d e_{t-m}}{d B_{k m i}^{\prime}} \\
& \quad\left(\mathrm{k}, \mathrm{k}^{\prime}=1,2, \mathrm{e}, \mathrm{r} ; \mathrm{k}_{\mathrm{i}}=1,2, \mathrm{e}, \mathrm{r} ; \mathrm{m}_{\mathrm{i}}=1,2, \mathrm{e}, \mathrm{s}\right) \\
& \left.W_{j}(t)=\sum_{j=1}^{s} B_{i j} X_{t-j} . \mathrm{e}, \mathrm{s}\right) \\
& \frac{d e_{t}}{d G_{i}}=0, \frac{d^{2} e_{t}}{d G_{i} d G_{j}}=0,(\mathrm{i}, \mathrm{j}=1,2, \text { é , } \mathrm{R} ; \mathrm{t}=1,2, \text { é , } \mathrm{m}-1)
\end{aligned}
$$

From $\mathrm{e}_{\mathrm{t}}=0 \quad(\mathrm{t}=1,2$, é , $\mathrm{m}-1), \frac{d e_{t}}{d G_{i}}=0, \frac{d^{2} e_{t}}{d G_{i} d G_{j}}=0, \quad$ and $\frac{d e_{t}}{d B_{k m i}}+\sum_{j=1}^{s} W_{j}(t) \frac{d e_{t-j}}{d B_{k m i}}=-X_{t-k} e_{t-m} \quad\left(\mathrm{k}=1,2\right.$,é ,r $; \mathrm{m}_{\mathrm{i}}$ $=1,2$,é ,s), it follows that the second order derivatives with respect to $\psi_{i}\left(\mathrm{i}=0,1,2\right.$, é , p) and $\theta_{i} \quad(\mathrm{i}=0,1,2$, é , q) are zero. For a given set of values $\left\{\psi_{i}\right\}$ and $\left\{\mathrm{B}_{\mathrm{ij}}\right\}$ one can evaluate the first and second order derivatives using the recursive equations 6,7 and 10 .

Let $\quad V(G)=\frac{d Q(G)}{d G_{1}}, \frac{d Q(G)}{d G_{2}}, \ldots \ldots . ., \frac{d Q(G)}{d G_{R}}$

and let $H(G)=\left[d^{2} Q(G) / d G_{i} d G_{j}\right]$ be a matrix of second partial derivatives. Expanding $V(\mathbf{G})$, near $G=\dot{G}$ in a Taylor series, we obtain $[V(G)]_{\breve{G}_{=G}}=0=V(G)+H(G)(\breve{G}-G)$. Rewriting this equation, we have $\dot{G}-G=-H^{-1}(G) V(G)$, thereby obtaining an iterative equation given by $G^{(k+1)}=G^{(k)}-H^{-1}\left(G^{(k)}\right) V\left(G^{(k)}\right)$, where $G^{(k)}$ is the set of estimates obtained at the $k^{\text {th }}$ stage of iteration. The estimates obtained by the above iterative equations usually converge. For starting the iteration, we need to have good sets of initial values of the parameters. This is done by fitting the best subset of the linear part of the bilinear model.

\section{Predictive Performance of the Models}

In order to compare the performance of the bilinear models, it is necessary that we should obtain the forecasts and these are obtained as follows:

Suppose $\left\{\mathrm{X}_{\mathrm{t}}\right\}$ is a discrete time series and we wish to predict $X_{t_{0}+h}$ given the semi-infinite realization $\left(X_{s}, s \leq t_{0}\right)$. Let the predictor be $\tilde{X}_{t_{0}}(h)$. Then it is well known that $E\left[X_{t_{0}+h}-\tilde{X}_{t_{0}}(h)\right]^{2}$ is minimum if and only if $\tilde{X}_{t_{0}}(h)=E\left(X_{t_{0}+h} / X_{s} s \leq t_{0}\right)$. The evaluation of $\tilde{X}_{t_{0}}(h)$ from the model depends on the unknown parameters. 
Typically, we substitute the least squares estimates of these parameters, and then calculate the predictors. The predictors thus obtained are denoted by $\tilde{X}_{t_{0}}(h),(\mathrm{h}=1,2$, é $)$ and the error by $\breve{\bar{E}_{t_{0}}}(h)=X_{t_{0}+h}-\breve{\bar{X}}_{t_{0}}(h)$ and the mean sum of squares of the errors of the predictors for the period $\left(\mathrm{t}_{0}+\mathrm{h}, \mathrm{t}_{\mathrm{o}}+\mathrm{h}\right.$ +1 , é , $\left.\mathrm{t}_{\mathrm{o}}+\mathrm{h}+\mathrm{M}\right)$ is $\quad \check{\mathscr{C}}_{\check{\underline{E}}}^{2}(h)=\frac{1}{M} \sum_{j=1}^{M} \breve{\vec{E}}_{t_{0}+j}(h)$

\section{RESULTS AND DISCUSSION}

To present the application of the model and its forecast, we will use a real time series dataset, the Wolfer sunspot. The scientists track solar cycle by counting sunspots $i \overrightarrow{~ c o o l ~ p l a n e t-s i z e d ~ a r e a s ~ o n ~ t h e ~ S u n ~ w h e r e ~ i n t e n s e ~}$

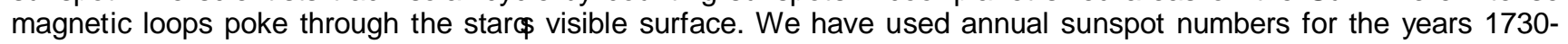
1879, giving 150 observations.

\section{Generalized Integrated Autoregressive Model}

Fitted Model at $\mathrm{t}=150$

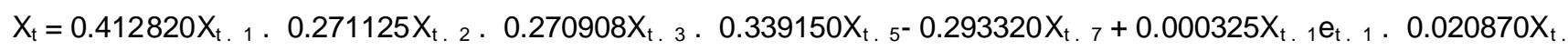

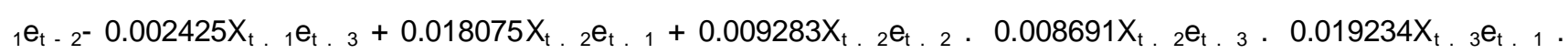
$0.007737 X_{t i ̈} e_{t i ~} 2+e_{t}$

\section{One-Dimensional Integrated Autoregressive Bilinear Time Series Model}

Fitted Model at $\mathrm{t}=150$

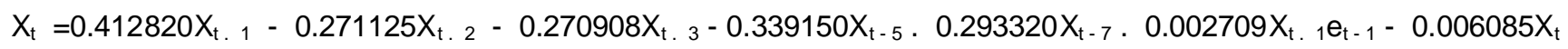

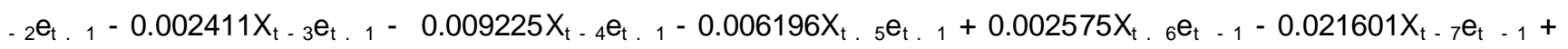

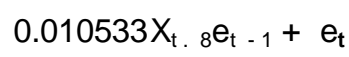

Table 1: Residual variance and mean squares error for forecast (sunspot data)

\begin{tabular}{|l|l|l|}
\hline MODEL & $\begin{array}{l}\text { One-Dimensional Integrated } \\
\text { Autoregressive Bilinear Model }\end{array}$ & $\begin{array}{l}\text { Generalized Integrated Autoregressive } \\
\text { Bilinear Model }\end{array}$ \\
\hline$\sigma_{e}^{2}$ & 207.50 & 193.20 \\
\hline MSEF & 15.55 & 14.28 \\
\hline
\end{tabular}

From Table 1, it is clear that the generalized integrated autoregressive bilinear time series model has smaller residual variance when compared with one-dimensional integrated autoregressive models. Also generalized integrated autoregressive bilinear time series model has the smaller mean squares error for the forecast when compared with one-dimensional integrated autoregressive time series models. And as a result the performance of generalized integrated autoregressive bilinear time series models is better when it is used for forecasting. 


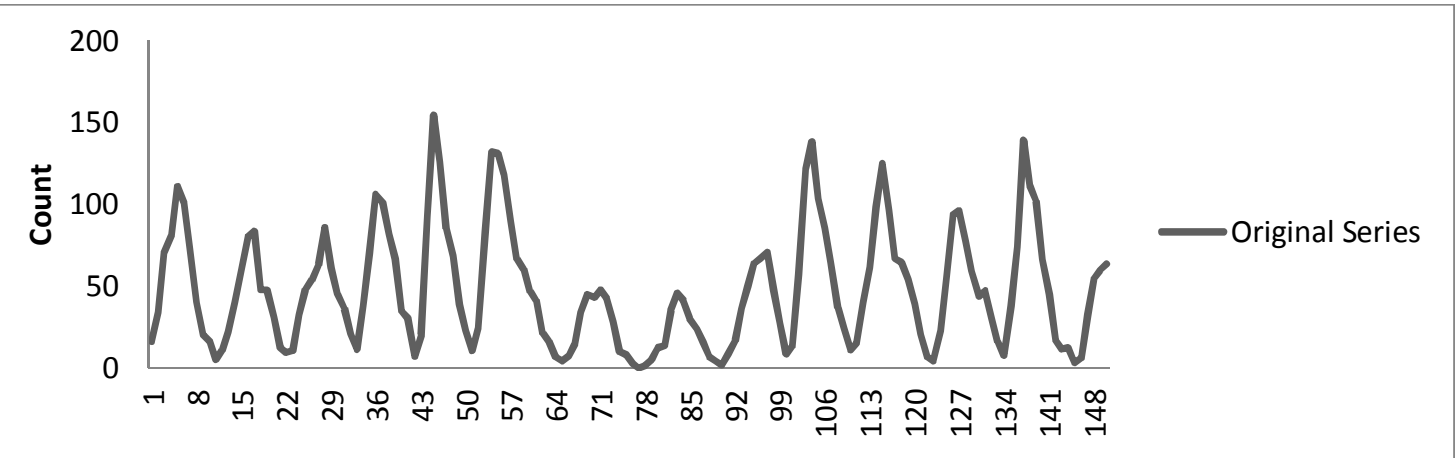

Figure 1: Time Plot of Sunspot Data (Original Series)

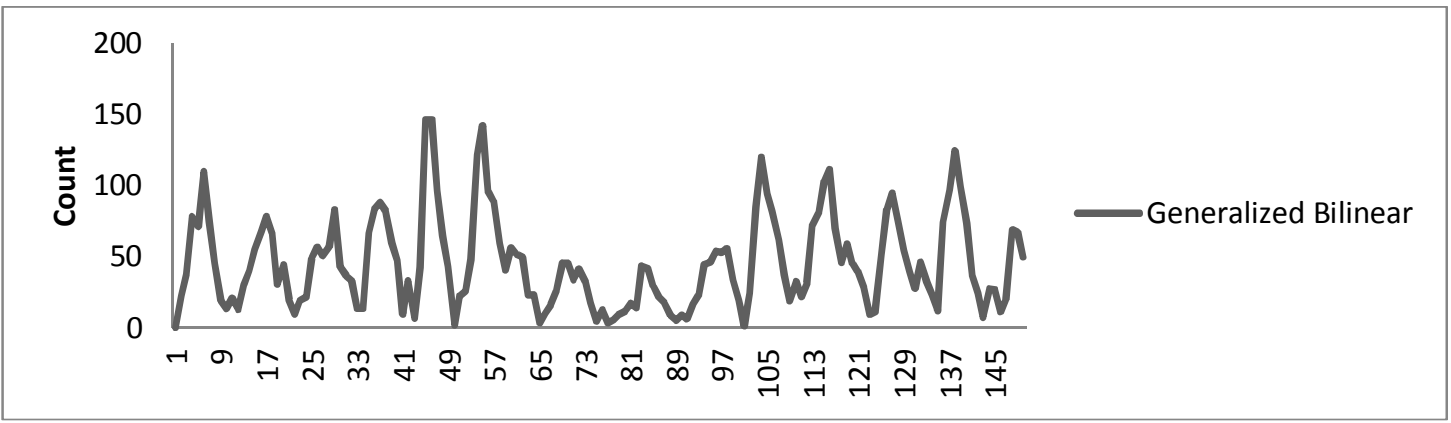

Figure 2: Time Plot of Forecast using Generalized Integrated Autoregressive Bilinear Model

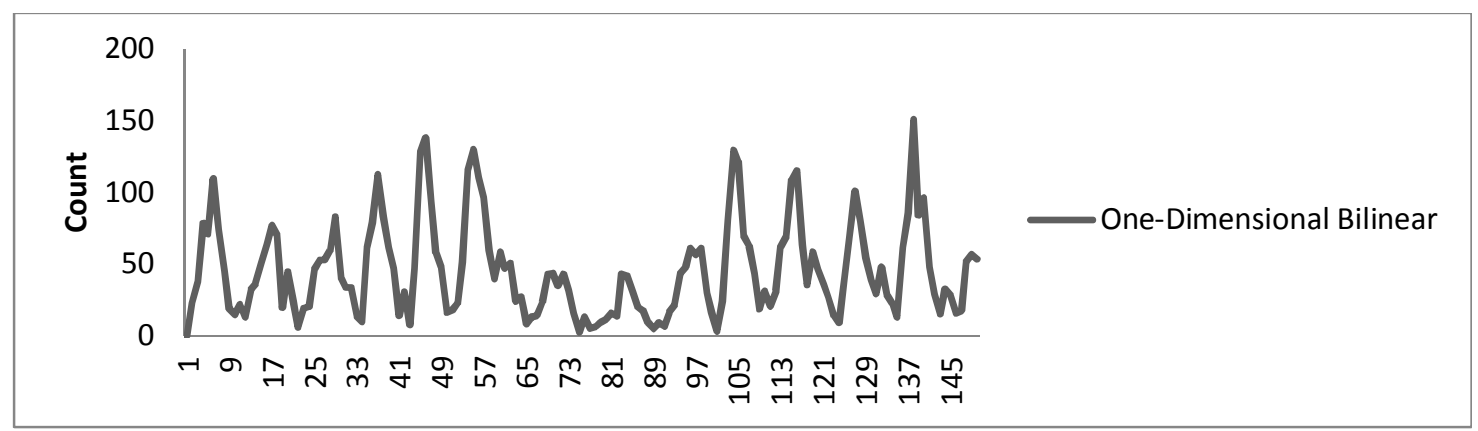

Figure 3: Time Plot of Forecast using One-Dimensional Integrated Autoregressive Bilinear Model 


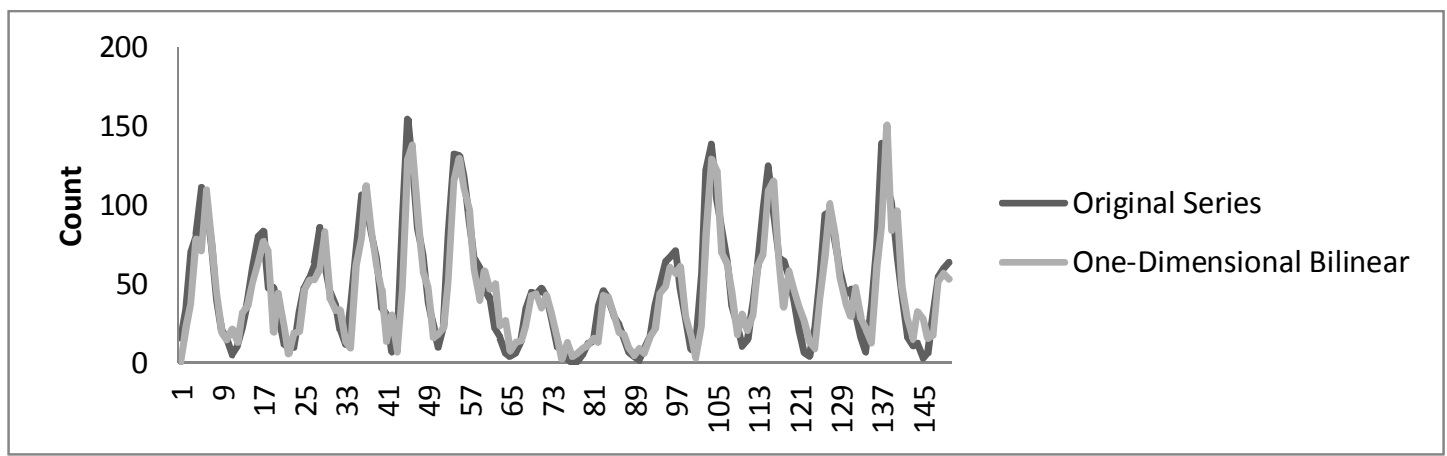

Figure 4: Time Plot of Forecast using Original Series and One-Dimensional Integrated Autoregressive Bilinear Model

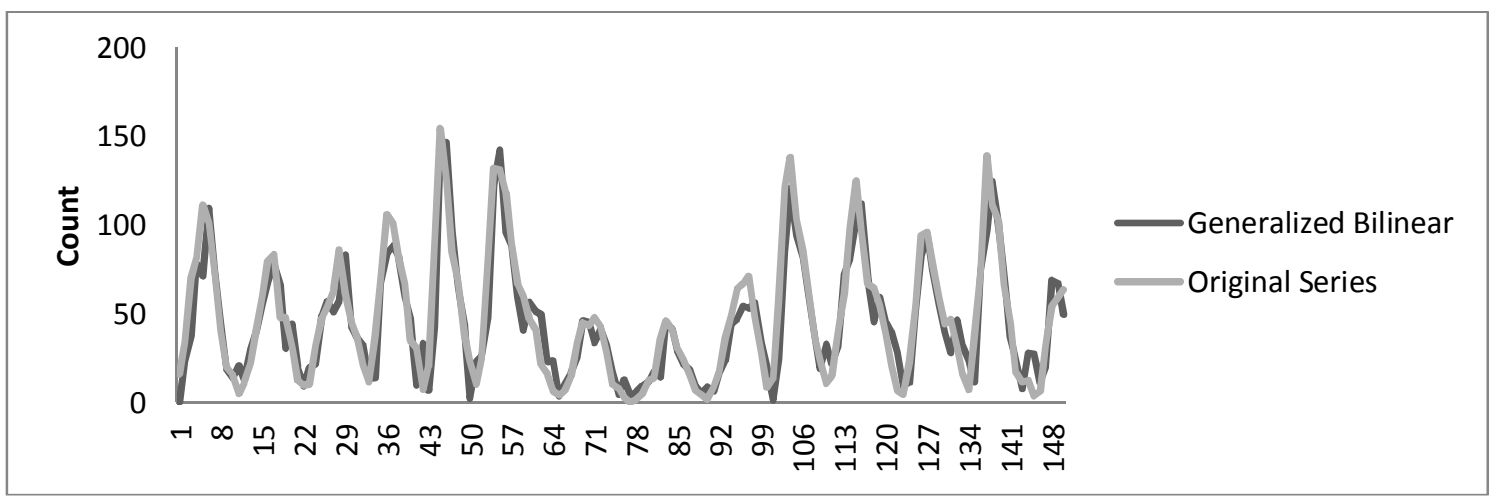

Figure 5: Time Plot of Forecast using Original Series and Generalized Integrated Autoregressive Bilinear Model

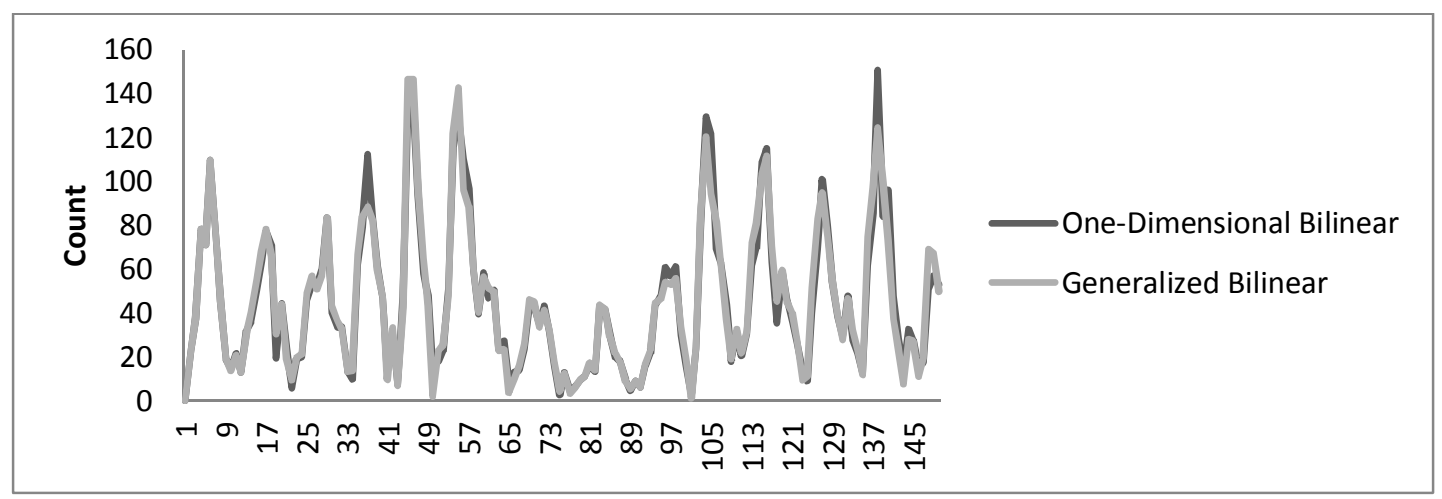

Figure 6: Time Plot of Forecast using Generalized and One-Dimensional Integrated Autoregressive Bilinear Models 


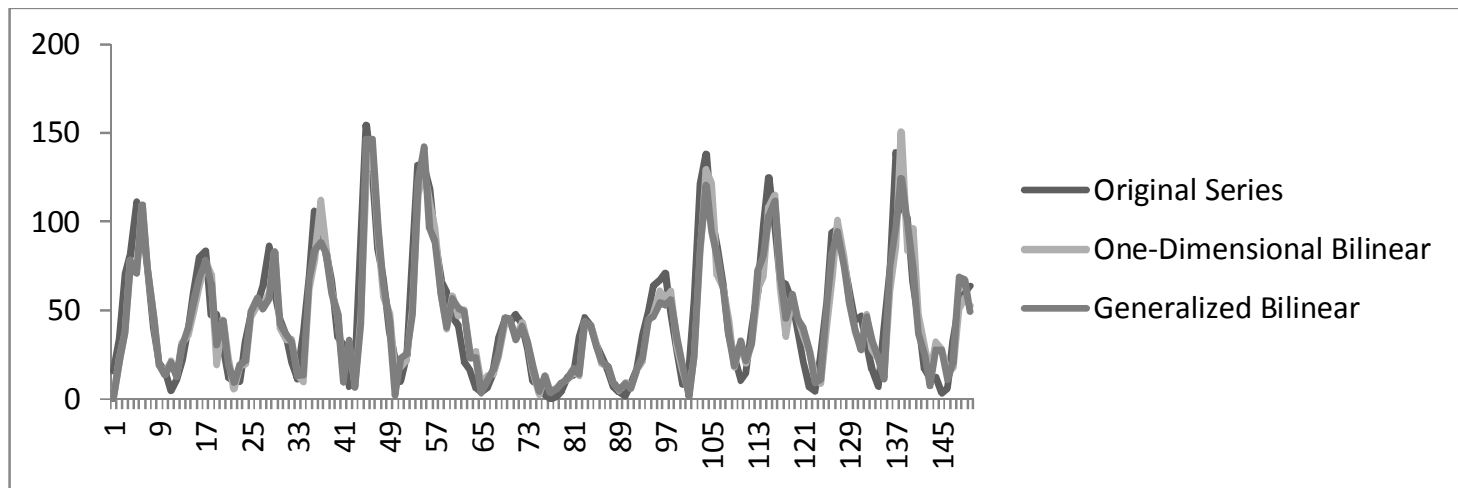

Figure 7: Time Plot of Original Series and Forecast using Generalized and One-Dimensional Integrated Autoregressive Bilinear Models

Figure 1 shows the graph of original series. Figure 2 shows graph of forecasts of generalized model while Figure 3 shows graph of forecast of one-dimensional model. Figures 4 and 5 compare graph of original series with graph of forecast of one-dimensional and generalized models while figure 5 compares graph of one-dimensional and generalized models. Figure 7 compares graph of original series and graph of forecast of one-dimensional and generalized models together.

\section{CONCLUSION}

Two bilinear time series models that were capable of achieving stationary for all non linear series were considered. These two models were used to forecast the future value having estimated their parameters. Generalized integrated autoregressive model outperformed one dimensional integrated autoregressive model after we have studied the residual variance attached to the two models. The mean square error for forecast for the models were studied and we found out that the mean square error attached to generalized bilinear model was smaller than one dimensional model. The two models were used to forecast. On the basis of the forecasting performance, generalized integrated autoregressive bilinear time series model formed a useful class of non-linear model for forecasting.

\section{REFERENCES}

Boonaick, K. Stensholt, B. K. and Stensholt E., 2005. Multivariate Bilinear Time Series: A Stochastic Alternative in Population Dynamics. Geophysical Research Abstracts. Volume 7, 02219 @Europeans Geosciences Union 2005.

Cathy, W. S., 1997. Detection of Additive Outliers in Bilinear Time Series. Computational Statistics and Data Analysis 24, 283-294
Doukhan, P., Latour, A., and Oraichi, D., 2006. A Simple integerï Valued Bilinear Time Series Model. Advanced Applied Probability. 38, 559-578.

Drost, F. C., Akker, R. V. and Werker, B. J. M., 2007. Note on Integerï Valued Bilinear Time Series Models. Econometrics and Finance group Center. ISSN 0924-7815, Tilburg University, the Netherlands.

Gabr, M. M. and Subba Rao, T., 1981. The Estimation and Prediction of Subset Bilinear Time Series Models with Application. Journal of Time Series Analysis. 2(3), 89-100.

Gonclaves, E., Jacob P. and Mendes-Lopes, N., 2000. A Decision Procedure for Bilinear Time Series Based on the Asymptotic Separation. Statistics, 333-348.

Liu, J., 1992. On stationarity and Asymptotic Inference of Bilinear time series models. Statistica Sinica Vol. 2. No. 2. 479-494.

Ojo J. F., 2009. The Estimation and Prediction of Autoregressive Moving Average Bilinear Time Series Models with Applications. Global Journal of Mathematics and Statistics. 1(2), 111-117.

Ojo, J. F and Shangodoyin, D. K., 2010. Theory of Generalized Integrated Autoregressive Bilinear Time series Modelling. Journal of Engineering and Applied Sciences. Volume 2, 53-61.

Ojo, J. F., 2011. On the Theory of One-Dimensional Integrated Autoregressive Bilinear Time Series Models. Ife Journal of Science. 13(1), 209-217. 
Pham, T. D. and Tran, L. T., 1981. On the First Order Bilinear Time Series Model. Jour. Appli. Probability. 18, 617-627.

Rao, M. B., Rao, T. S. and Walker, A. M., 1983. On the Existence of some Bilinear Time Series Models. Journal of Time Series Analysis. 4(2), 60-76.

Shangodoyin D. K. and Ojo J. F., 2003. On the Determination and Estimation of Subset Stationary Bilinear Time Series Autoregressive
Models. Journal of Science, Engineering and Technology. Vol. 10, No 3 pages 5080-5091.

Subba Rao, T., 1981. On Theory of Bilinear time Series Models. Jour. R. Sta. Soc. B. 43, 244-255

Usoro, A. E. and Omekara, C. O., 2008. Lower Diagonal Bilinear Moving Average Vector Models. Advances in Applied Mathematical Analysis. 3(1), 49-54.

Wang H. B. and Wei B. C., 2004. Separable Lower Triangular Bilinear Model. J. Applied Probability. 41(1), 221-235. 